\title{
Validity and Agreement between the 28-Joint Disease Activity Score Based on C-Reactive Protein and Erythrocyte Sedimentation Rate in Patients with Rheumatoid Arthritis
}

\author{
Louise Nielung, ${ }^{1}$ Robin Christensen, ${ }^{1}$ Bente Danneskiold-Samsøe, ${ }^{1,2}$ Henning Bliddal, ${ }^{1,2}$ \\ Christian Cato Holm, ${ }^{1}$ Karen Ellegaard, ${ }^{1}$ Hanne Slott Jensen, ${ }^{3}$ and Else Marie Bartels ${ }^{1}$ \\ ${ }^{1}$ The Parker Institute, Department of Rheumatology, Copenhagen University Hospital, 2000 Frederiksberg, Denmark \\ ${ }^{2}$ Department of Clinical Medicine, Faculty of Health and Medical Sciences, University of Copenhagen, 2200 Copenhagen N, Denmark \\ ${ }^{3}$ Department of Rheumatology, Copenhagen University Hospital, 2000 Frederiksberg, Denmark
}

Correspondence should be addressed to Else Marie Bartels; else.marie.bartels@regionh.dk

Received 10 November 2014; Revised 18 December 2014; Accepted 19 December 2014

Academic Editor: Burkhard Leeb

Copyright (C) 2015 Louise Nielung et al. This is an open access article distributed under the Creative Commons Attribution License, which permits unrestricted use, distribution, and reproduction in any medium, provided the original work is properly cited.

\begin{abstract}
Objective. To validate the agreement between the 28-joint disease activity score based on erythrocyte sedimentation rate (DAS28ESR) and the 28-joint disease activity score based on C-reactive protein (DAS28-CRP) in a group of Danish patients with rheumatoid arthritis (RA). Methods. Data from 109 Danish RA patients initiating biologic treatment were analysed at baseline and following one year of treatment. Participants were retrospectively enrolled from a previous cohort study and were considered eligible for this project if CRP and ESR were measured at baseline and at the follow-up visit. To assess the extent of agreement between the two DAS28 definitions, the "European League Against Rheumatism" (EULAR) response criteria based on each definition were calculated with cross-classification. Weighted Kappa $(\kappa)$ coefficients were calculated, and Bland-Altman plots were used to illustrate degree of agreement between DAS28 definitions. Results. The 75 eligible patients were classified as EULAR good, moderate, and nonresponders with good agreement (61/75; 81\%) between DAS28-CRP and DAS28-ESR ( $\kappa=0.75$ (95\% CI: 0.63 to 0.88 )). Conclusions. According to our findings, DAS28-CRP and DAS28-ESR are interchangeable when assessing RA patients and the two versions of DAS28 are comparable between studies.
\end{abstract}

\section{Introduction}

Rheumatoid arthritis (RA) is a common inflammatory disease characterised by poly-articular inflammation of the synovial tissue [1]. The disease activity score (DAS) is a tool used to monitor disease activity in RA. DAS combines tender and swollen joint counts, an inflammatory marker, and a patient-reported measure of general health [2]. The first DAS was based on an examination of 44 joints (DAS44) [2], and this was later followed by a reduced and simplified version based on 28 joints, DAS28 [3]. DAS28 is amongst the RA disease activity measures recommended by the American College of Rheumatology (ACR) [4].

DAS28 was originally using the erythrocyte sedimentation rate (ESR) as the inflammation marker and named DAS28-ESR. DAS28-ESR was further extensively validated for its use in clinical trials $[3,5,6]$. Later Fransen et al. suggested an alternative formulation of DAS28 based on Creactive protein (DAS28-CRP) [7], since CRP is a preferential measure of inflammation compared to ESR $[8,9]$, with ESR being confounded by age, sex, anaemia, time of day, plasma viscosity, and abnormal shape and size of the red blood cells [8].

Previously Wells et al. [10] compared DAS28-CRP with DAS28-ESR. They concluded that while the DAS28-CRP yielded a better EULAR response [11] more often than the DAS28-ESR, the validation profile was similar to the DAS28ESR, indicating that both measures are useful when assessing disease activity in patients with RA [10]. Agreement between the two DAS28 in classification into high and moderate disease activity has though been questioned by Hensor et al. [12]. 
DAS28 is at present one of the recommended and most widely used composite measures in rheumatology, both in clinical trials and when monitoring RA patients in daily clinical practice. Due to the extent use of DAS28, it is important to determine if DAS28-CRP and DAS28-ESR are interchangeable, or to which degree they are comparable.

This study aims at validating the agreement between the two DAS28 scores in assessment of a group of Danish patients with RA prior to and following a year of treatment with anti-TNF- $\alpha$ biologics.

\section{Methods}

2.1. Datasets. A cohort of 109 Danish RA patients from the Rheumatology Clinic, Bispeberg and Frederiksberg Hospital, initiating treatment with a biological agent, were studied prior to and following one year of treatment. All patients were registered in the Danish DANBIO registry [13] and were enrolled in a previously published Danish cohort study [14] approved by the Biomedical Research Ethics Committee for the Capital Region of Denmark (KF01-045/03).

At both visits the patients were seen by a rheumatologist who assessed the number of swollen joints (SJC) and the number of tender joints (TJC). Blood samples were taken at the Clinical Chemistry Department at Bispebjerg and Frederiksberg Hospital to asses CRP and ESR. CRP was measured in heparin plasma with immunoturbidimetric absorption photometry (Roche/Hitachi cobas-Csystems, Roche Diagnostics GmbH, D-68298 Mannheim), with a value $\leq 10 \mathrm{mg} / \mathrm{L}$ being considered normal concentration, detection limit $0.3 \mathrm{mg} / \mathrm{L}$. ESR was measured according to the original Westergren's method [15]. Patient-reported general health $(\mathrm{PtGH})$ was assessed on a visual analogue scale (VAS) ranging from 0 to $100 \mathrm{~mm}$, with $0=$ best and $100=$ worst. The patients were treated with adalimumab, etanercept, or infliximab and were all fulfilling the American College of Rheumatology (ACR) criteria for the diagnosis of RA [1].

2.2. Measures of Disease Activity and Criterion Validity (DAS28). DAS28 is calculated by using the following formula based on TJC, SJC, PtGH, and either CRP (mg/L) or ESR $(\mathrm{mm} / \mathrm{h})$ :

$$
\begin{aligned}
& \text { DAS28-CRP }=0.56 \cdot \sqrt{\mathrm{TJC} 28}+0.28 \cdot \sqrt{\mathrm{SJC} 28}+0.014 \cdot \\
& \mathrm{PtGH}+0.36 \cdot \ln (\mathrm{CRP})+0.96[10] \\
& \mathrm{DAS} 28-\mathrm{ESR}=0.56 \cdot \sqrt{\mathrm{TJC} 28}+0.28 \cdot \sqrt{\mathrm{SJC} 28}+0.014 \cdot \\
& \mathrm{PtGH}+0.70 \cdot \ln (\mathrm{ESR})[3] .
\end{aligned}
$$

The RA disease activity level is defined as low (DAS28 $\leq$ $3.2)$, moderate $(3.2<\mathrm{DAS} 28 \leq 5.1)$, or high $(\mathrm{DAS} 28>5.1)$ [11].

In the present study, the disease activity scores were calculated at baseline and again one year later to compare the improvement within the "European League Against Rheumatism" (EULAR) response criteria which were classified according to Fransen and Van Riel [11].

Good responders: improvement $>1.2$, and a present DAS28 $\leq 3.2$.
Moderate responders: improvement $>0.6$ to $\leq 1.2$, and a present DAS28 $\leq 5.1$; or improvement $>1.2$, and a present DAS28 > 3.2.

Nonresponders: improvement $\leq 0.6$, or improvement $>$ 0.6 to $\leq 1.2$, and a present DAS2 $>5.1$.

To accomplish remission, the patients had to have a DAS28 < 2.6. The EULAR response was calculated for all patients available at the one-year assessment, with the purpose of cross-classification for both DAS28 definitions to validate and compare the two definitions.

2.3. Statistical Analysis. For the quantification of reproducibility between the disease measures, two types of analyses were applied: the weighted Kappa statistics for criteria agreement and the Bland and Altman method for assessing agreement [16]. A priori Kappa was defined as a value between 0.60 and 0.80 to indicate good agreement between the scores [17]. The Bland and Altman method provides insight into the distribution of differences between observers. It presents the size, direction, and range of differences between DAS28 observations in the same units. The agreement was quantified by calculating the mean difference $(d)$ between the two DAS28 observations and the standard deviation (SD) for this difference. The closer $d$ is to zero, and the smaller the SD is of this difference, the better the agreement between DAS28 indices is. Differences between the two observations were plotted against the average of the two measurements. The 95\% limits of agreement were defined as the mean difference between the observations \pm 1.96 . SD of the differences, indicating the total error (bias and random error together).

\section{Results}

Of the 109 patients in the study of Ellegaard et al. [14], 108 patients had CRP and ESR values from their first visit. Following one year of treatment, 75 were still in therapy with the biological agents, and it was possible to determine DAS28 based on either CRP or ESR. Demographics and patients characteristics are seen in Table 1. Of the 75 follow-up patients, the median age was 59.6 years, the median disease duration was 6 years, and the patient population consisted primarily of females $(73 \%)$.

The patients were classified as good, moderate, and nonresponders according to their DAS28 improvement after one year. We found a good agreement between the response indices based on DAS28-CRP and DAS28-ESR with a weighted $\kappa$ of 0.75 (95\% CI: 0.63 to 0.88 ). The correlation between the two DAS28 is shown in Table 2. The absolute agreement between DAS28-CRP and DAS28-ESR was 81\% (61/75).

Using Bland-Altman plots to illustrate the agreement between DAS28-CRP and DAS28-ESR gave a similar answer. Figure 1(a) shows DAS28 values of the patients at baseline and it can be seen that most of the observations are lying between the mean and $\pm 1.96 \times \mathrm{SD}$. The mean difference between the two definitions is -0.32 (limits of agreement: -1.05 to 0.40 ). Figure 1(b) shows change from baseline assessed after one year. The mean difference was -0.09 (limits of agreement: -0.70 to 0.52 ). 


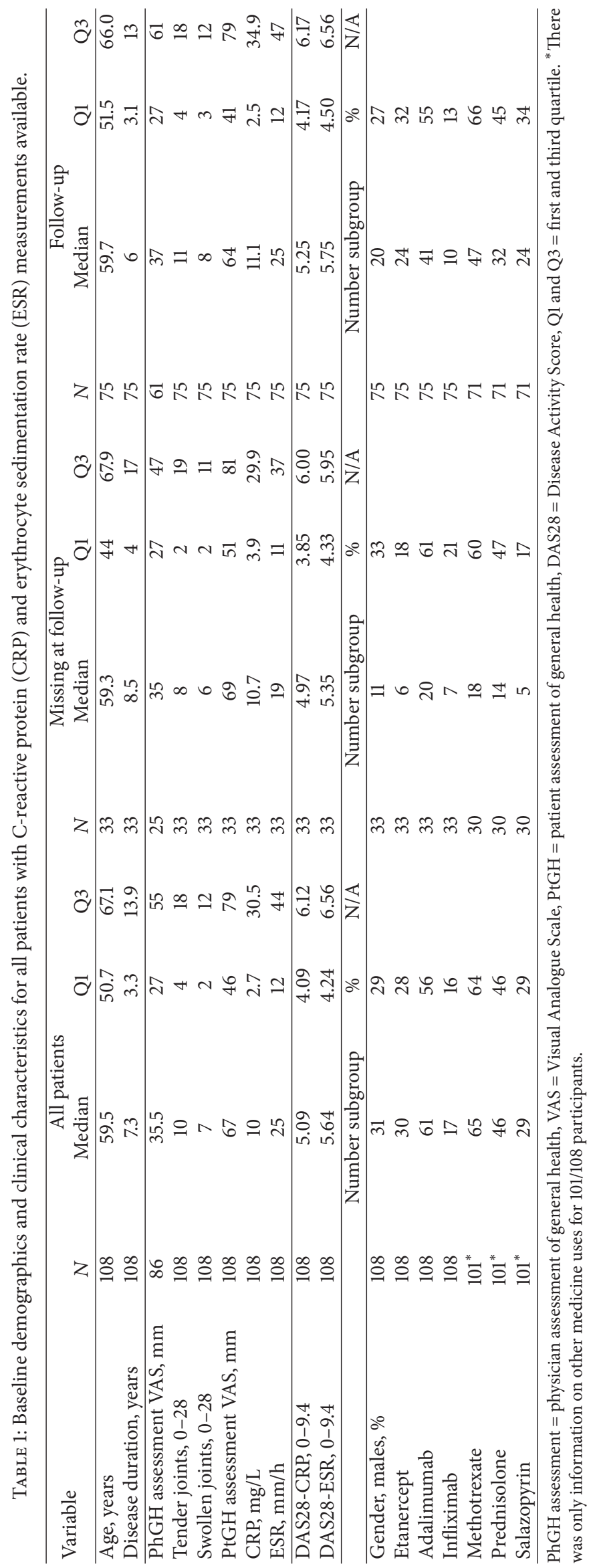


TABLE 2: Cross-classification of patients at low, moderate, or high disease activity when using DAS28-ESR versus DAS28-CRP.

\begin{tabular}{|c|c|c|c|c|}
\hline \multirow{2}{*}{ Baseline } & & \multicolumn{3}{|c|}{ DAS28-ESR } \\
\hline & & Low disease activity (\%) & Moderate disease activity (\%) & High disease activity (\%) \\
\hline \multirow{3}{*}{ DAS28-CRP } & Low disease activity (\%) & $5(6.7)$ & $2(2.7)$ & $0(0)$ \\
\hline & Moderate disease (\%) activity & $1(1.3)$ & $14(18.7)$ & $12(16)$ \\
\hline & High disease activity (\%) & $0(0)$ & $1(1.3)$ & $40(53.3)$ \\
\hline \multirow{2}{*}{ One year follow-up } & & \multicolumn{3}{|c|}{ DAS28-ESR } \\
\hline & & Low disease activity (\%) & Moderate disease activity (\%) & High disease activity (\%) \\
\hline \multirow{3}{*}{ DAS28-CRP } & Low disease activity (\%) & $36(48)$ & $10(13.3)$ & $0(0)$ \\
\hline & Moderate disease activity (\%) & $0(0)$ & $24(32)$ & $3(4)$ \\
\hline & High disease activity (\%) & $0(0)$ & $0(0)$ & $2(2.7)$ \\
\hline
\end{tabular}

$n=75$, patients with ESR and CPR measures available at baseline and at the follow-up visit one year later.

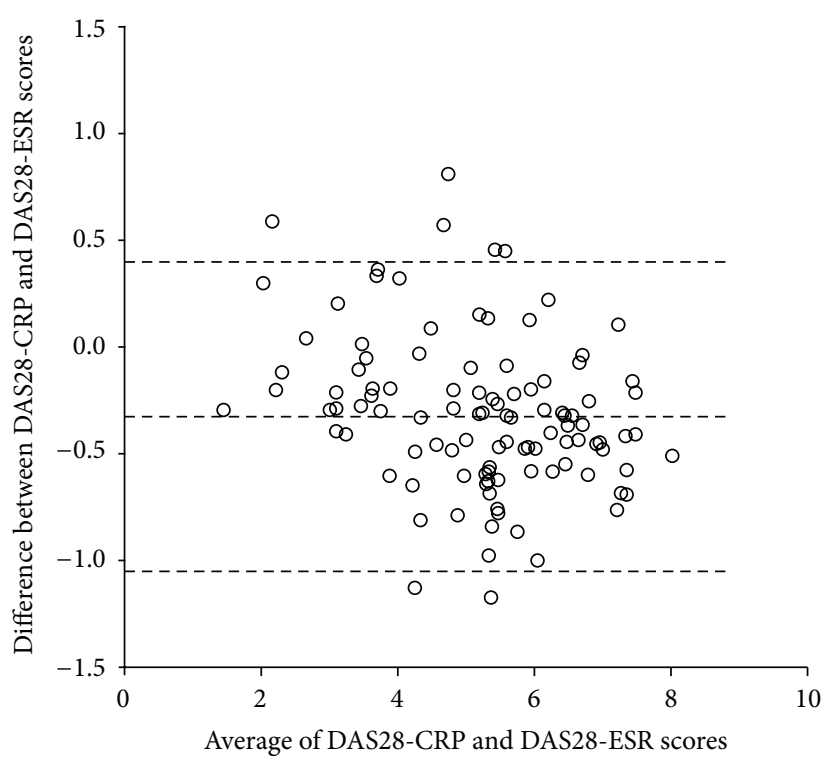

(a)

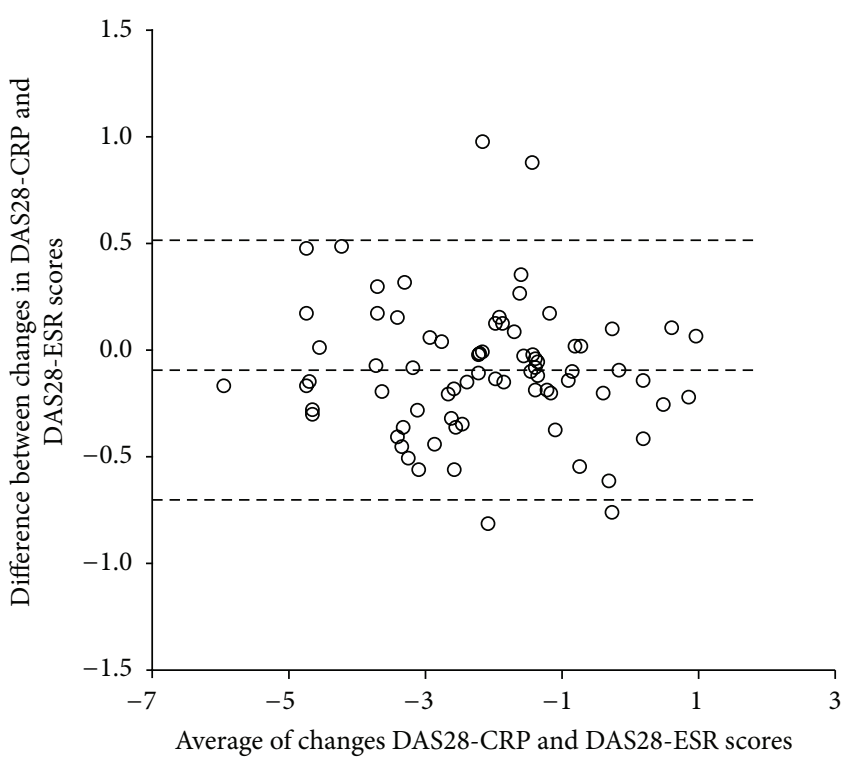

(b)

FIGURE 1: (a) Bland-Altman plot of disease activity score (DAS28) C-reactive protein (CRP) and DAS28 erythrocyte sedimentation rate (ESR) values at baseline. Difference between DAS28-CRP and DAS28-ERS scores versus mean value of DAS28-CRP and DAS28-ESR at baseline is shown. The mean difference is represented by the central line and the upper and lower bound represent $\pm 1.96 \mathrm{SD}$ from the mean. (b) BlandAltman plot of the change in disease activity score (DAS28) C-reactive protein (CRP) and DAS28 erythrocyte sedimentation rate (ESR) values assessed after one year. Difference between changes in DAS28-CRP and DAS28-ESR scores versus mean value of the changes of DAS28-CRP and DAS28-ESR at baseline is shown. The mean difference is represented by the central line and the upper and lower bound represent $\pm 1.96 \mathrm{SD}$ from the mean.

Looking at the EULAR response, where there was a divergence between using DAS28-CRP and DAS28-ESR, 12 showed a better response (in terms of responder category) using DAS28-CRP, while two patients showed a better response using DAS28-ESR.

\section{Discussion}

This study compared the EULAR responder categories using DAS28-CRP and DAS28-ESR in 75 Danish RA patients in biologic treatment. According to our findings, it is in general possible to use either CRP or ESR in the calculation of DAS28. $81 \%$ of our patients were classified as having the same EULAR response (according to response category) regardless of using DAS28-CRP or DAS28-ESR, while 19\% would be allocated differently in terms of disease severity between the two expressions of DAS28.

Studies from Wells et al. [10] (758 patients), Inoue et al. [18] (6729 patients), and Siemons et al. [19] (682 patients) confirmed our data, concluding that DAS28-CRP and DAS28-ESR agree in general, but that DAS28-CRP may have a tendency to underestimate the disease activity. Matsui et al. [20] found, on the other hand, that the two versions of DAS28 could not replace each other in a large study of 3073 Japanese RA patients.

In our study, the 12 patients (9\%) with a better EULAR response when using DAS28-CRP compared to DAS28-ESR do not necessarily represent an underestimation of disease activity when using DAS28-CRP. In most of these cases, DAS28-ESR is $0.1-0.3$ from being in the same responder 
category as assessed by DAS28-CRP. The difference seen in our 12 patients could also be caused by the many factors affecting the ESR measurement, where presence of immunoglobulins like rheumatoid factor and/or of anticyclic citrullinated peptide antibodies (anti-CCP) could be the main culprit [21-24]. This is supported by that all 12 patients were rheumatoid-factor positive, and 8/12 were, furthermore, also anti-CCP positive. The effect is though small in our group. This is in agreement with the finding that the two DAS28 most often give the same classification and is in agreement with Radovits et al. [25].

With a wider use of CRP as a standard today, DAS28 will probably most frequently be calculated using CRP in the future, and one may consider if the DAS28-CRP cut-off points should be changed [26], or the DAS28-CRP definition should be modified [12], or if the differences between the two DAS28 in practice do have a clear trend justifying a change of cut-off points.

\section{Conclusion}

In conclusion, we have validated the use of DAS28-CRP with DAS28-ESR, and DAS28-CRP is in good agreement (81\%) with DAS28-ESR in our Danish group of RA patients, although DAS28-CRP may have a tendency to give a better EULAR response.

\section{Conflict of Interests}

All authors report no conflict of interests.

\section{Acknowledgment}

This study was supported by the Oak Foundation.

\section{References}

[1] F. C. Arnett, S. M. Edworthy, D. A. Bloch et al., "The American Rheumatism Association 1987 revised criteria for the classification of rheumatoid arthritis," Arthritis and Rheumatism, vol. 31, no. 3, pp. 315-324, 1988 .

[2] D. M. F. M. van der Heijde, M. A. van 't Hof, P. L. C. M. van Riel et al., "Judging disease activity in clinical practice in rheumatoid arthritis: first step in the development of a disease activity score," Annals of the Rheumatic Diseases, vol. 49, no. 11, pp. 916-920, 1990.

[3] M. L. L. Prevoo, M. A. van 'T Hof, H. H. Kuper, M. A. Van Leeuwen, L. B. A. Van De Putte, and P. L. C. M. Van Riel, "Modified disease activity scores that include twenty-eight-joint counts: development and validation in a prospective longitudinal study of patients with rheumatoid arthritis," Arthritis and Rheumatism, vol. 38, no. 1, pp. 44-48, 1995.

[4] J. Anderson, L. Caplan, J. Yazdany et al., "Rheumatoid arthritis disease activity measures: American college of rheumatology recommendations for use in clinical practice," Arthritis Care \& Research, vol. 64, no. 5, pp. 640-647, 2012.

[5] B. V. Cruyssen, S. van Looy, B. Wyns et al., "DAS28 best reflects the physician's clinical judgment of response to infliximab therapy in rheumatoid arthritis patients: validation of the DAS28 score in patients under infliximab treatment," Arthritis Research \& Therapy, vol. 7, pp. R1063-R1071, 2005.
[6] M. L. L. Prevoo, A. M. van Gestel, M. A. van 'T Hof, M. H. van Rijswijk, L. B. A. van de Putte, and P. L. C. M. van Riel, "Remission in a prospective study of patients with rheumatoid arthritis. American rheumatism association preliminary remission criteria in relation to the disease activity score," British Journal of Rheumatology, vol. 35, no. 11, pp. 1101-1105, 1996.

[7] J. Fransen, P. M. J. Welsing, R. M. H. De Keijzer, and P. L. C. M. Van Riel, "Disease activity scores using c-reactive protein: CRP may replace ESR in the assessment of RA disease activity," Annals of the Rheumatic Diseases, vol. 62, supplement 1, pp. 151158, 2003.

[8] I. Kushner, "C-reactive protein in rheumatology," Arthritis \& Rheumatism, vol. 34, no. 8, pp. 1065-1068, 1991.

[9] C.S. Crowson, M. U. Rahman, and E. L. Matteson, "Which measure of inflammation to use? A comparison of erythrocyte sedimentation rate and C-reactive protein measurements from randomized clinical trials of golimumab in rheumatoid arthritis," The Journal of Rheumatology, vol. 36, pp. 1606-1610, 2009.

[10] G. Wells, J.-C. Becker, J. Teng et al., "Validation of the 28-joint Disease Activity Score (DAS28) and European League Against Rheumatism response criteria based on C-reactive protein against disease progression in patients with rheumatoid arthritis, and comparison with the DAS28 based on erythrocyte sedimentation rate," Annals of the Rheumatic Diseases, vol. 68, no. 6, pp. 954-960, 2009.

[11] J. Fransen and P. L. C. M. Van Riel, "The Disease Activity Score and the EULAR response criteria," Clinical and Experimental Rheumatology, vol. 23, supplement 39, no. 5, pp. S93-S99, 2005.

[12] E. M. A. Hensor, P. Emery, S. J. Bingham, and P. G. Conaghan, "Discrepancies in categorizing rheumatoid arthritis patients by DAS-28(ESR) and DAS-28(CRP): can they be reduced?" Rheumatology, vol. 49, no. 8, Article ID keq117, pp. 1521-1529, 2010.

[13] M. L. Hetland, "DANBIO-powerful research database and electronic patient record," Rheumatology, vol. 50, no. 1, pp. 6977, 2011.

[14] K. Ellegaard, R. Christensen, S. Torp-Pedersen et al., "Ultrasound Doppler measurements predict success of treatment with anti-TNF- $\alpha$ drug in patients with rheumatoid arthritis: a prospective cohort study," Rheumatology, vol. 50, no. 3, pp. 506$512,2011$.

[15] "ICSH recommendations for measurement of erythrocyte sedimentation rate. International Council for Standardization in Haematology (Expert Panel on Blood Rheology)," Journal of Clinical Pathology, vol. 46, no. 3, pp. 198-203, 1993.

[16] J. M. Bland and D. G. Altman, "Agreed statistics: measurement method comparison," Anesthesiology, vol. 116, no. 1, pp. 182-185, 2012.

[17] J. R. Landis and G. G. Koch, "The measurement of observer agreement for categorical data," Biometrics, vol. 33, no. 1, pp. 159-174, 1977.

[18] E. Inoue, H. Yamanaka, M. Hara, T. Tomatsu, and N. Kamatani, "Comparison of Disease Activity Score (DAS)28- erythrocyte sedimentation rate and DAS28- C-reactive protein threshold values," Annals of the Rheumatic Diseases, vol. 66, no. 3, pp. 407409, 2007.

[19] L. Siemons, H. E. Vonkeman, P. M. ten Klooster, P. L. C. M. van Riel, and M. A. F. J. van de Laar, "Interchangeability of 28joint disease activity scores using the erythrocyte sedimentation rate or the C-reactive protein as inflammatory marker," Clinical Rheumatology, vol. 33, no. 6, pp. 783-789, 2014. 
[20] T. Matsui, Y. Kuga, A. Kaneko et al., "Disease Activity Score 28 (DAS28) using C-reactive protein underestimates disease activity and overestimates EULAR response criteria compared with DAS28 using erythrocyte sedimentation rate in a large observational cohort of rheumatoid arthritis patients in Japan," Annals of the Rheumatic Diseases, vol. 66, no. 9, pp. 1221-1226, 2007.

[21] J. Lyngbye, A. Kjær, S. A. Ladefoged, and P. H. Nissen, Lyngbyes Laboratoriemedicin, Nyt Nordisk Forlag, Copenhagen, Denmark, 2010.

[22] R. A. Crockson and A. P. Crockson, "Relationship of the erythrocyte sedimentation rate to viscosity and plasma proteins in rheumatoid arthritis," Annals of the Rheumatic Diseases, vol. 33, no. 1, pp. 53-56, 1974.

[23] M. T. M. Raijmakers, P. H. M. Kuijper, D. L. Bakkeren, and H. L. Vader, "The effect of paraproteins on the erythrocyte sedimentation rate: a comparison between the StarrSed and TEST 1," Annals of Clinical Biochemistry, vol. 45, no. 6, pp. 593-597, 2008.

[24] G. Mouterde, C. Lukas, P. Goupille et al., "Association of anticyclic citrullinated peptide antibodies and/or rheumatoid factor status and clinical presentation in early arthritis: results from the ESPOIR cohort," The Journal of Rheumatology, vol. 41, no. 8, pp. 1614-1622, 2014.

[25] B. J. Radovits, J. Fransen, P. L. C. M. van Riel, and R. F. J. M. Laan, "Influence of age and gender on the 28-joint Disease Activity Score (DAS28) in rheumatoid arthritis," Annals of the Rheumatic Diseases, vol. 67, no. 8, pp. 1127-1131, 2008.

[26] H. Mäkinen, H. Kautiainen, P. Hannonen, and T. Sokka, "Is DAS28 an appropriate tool to assess remission in rheumatoid arthritis?" Annals of the Rheumatic Diseases, vol. 64, no. 10, pp. 1410-1413, 2005. 


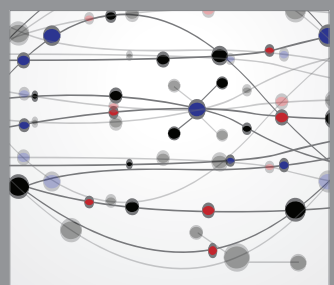

The Scientific World Journal
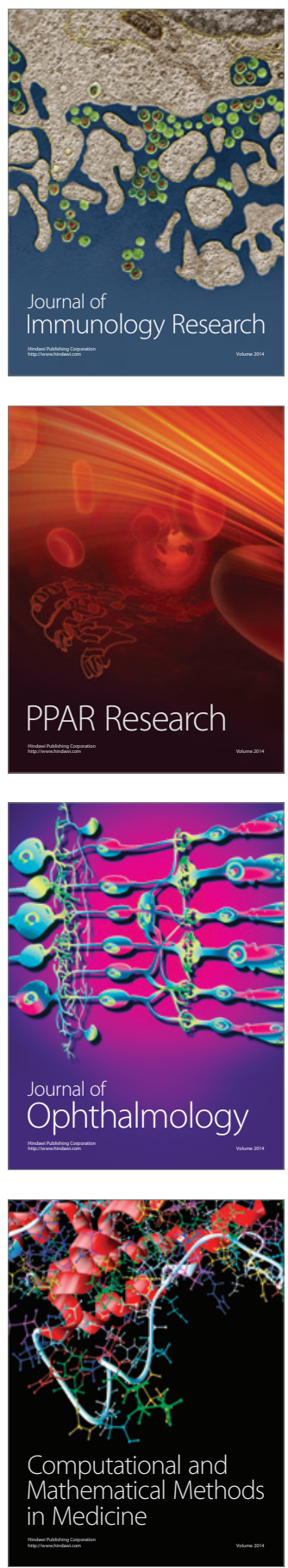

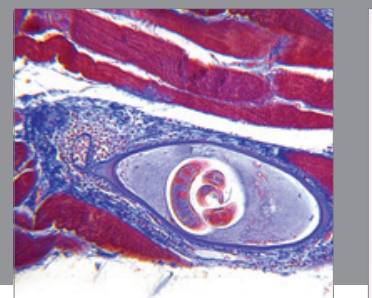

Gastroenterology

Research and Practice
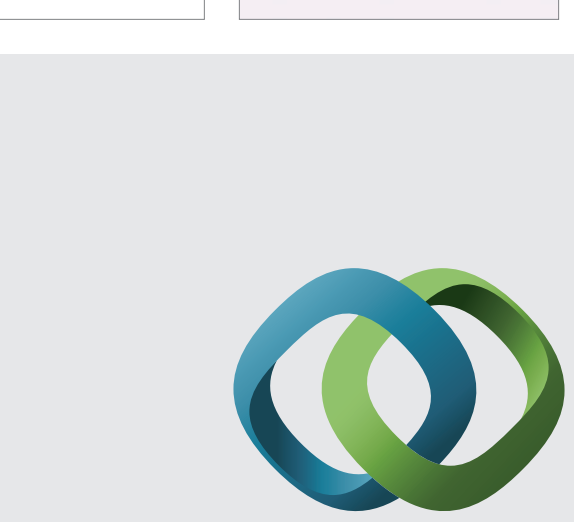

\section{Hindawi}

Submit your manuscripts at

http://www.hindawi.com
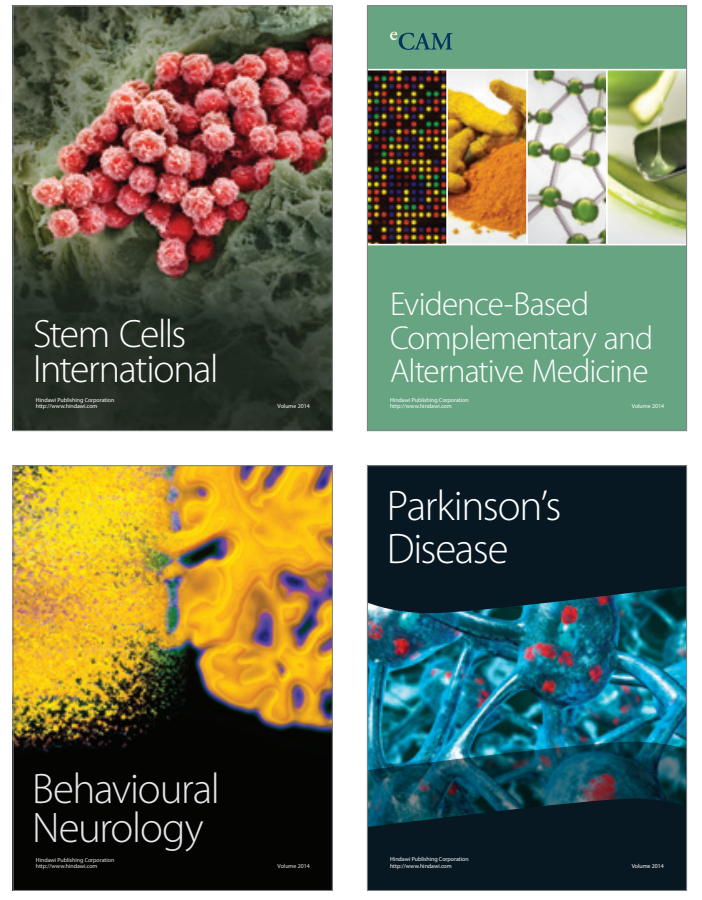
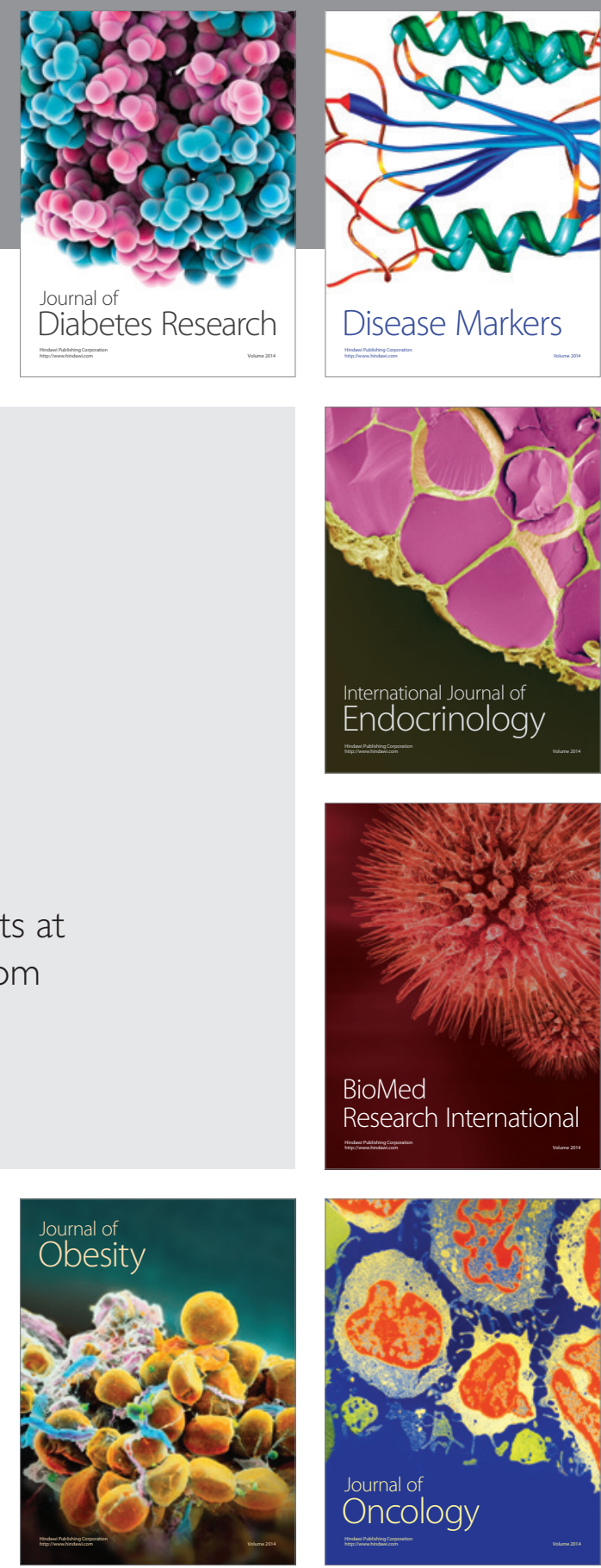

Disease Markers
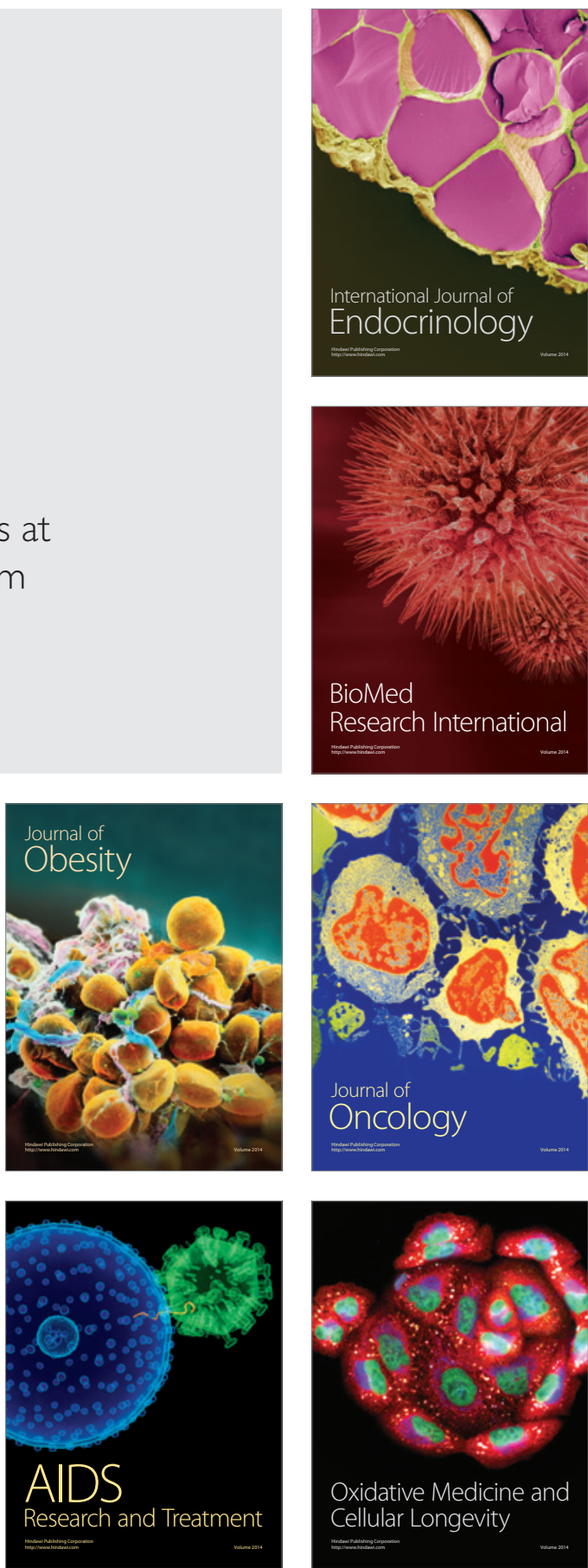